\title{
COMPLEX PROBABILITIES AND THE LANGEVIN EQUATION
}

\author{
Herbert W. HAMBER and Hai-cang REN \\ The Institute for Advanced Study, Princeton, NJ 08540, USA
}

Received 30 April 1985

\begin{abstract}
Complex probabilities arise in quantum systems where the euclidean action is complex, either because the couplings, or the field variables, are complex. Stochastic quantization using the Langevin equation allows one to study such systems, even though the probabilistic interpretation of the euclidean functional integral measure breaks down. The convergence of long time averages computed with the Langevin equation to the analytic continuation of the functional integral averages is investigated for some simple models. The proposed methods have a variety of applications, including nonperturbative studies of chiral gauge theories on the lattice.
\end{abstract}

It has recently been realized [1] that the Langevin equation of non-equilibrium statistical mechanics also represents an alternative to the path integral and canonical quantization procedures in field theory. The complex phase factor associated with a path in the functional integral formulation corresponds in statistical mechanics to the Boltzmann factor, which in turn has a probabilistic interpretation. If the quantum theory is considered in its Wick-rotated form, then the complex phase factor can usually be considered as a real probability amplitude. This has led to a fruitful application of a variety of stochastic methods to quantum field theories, which have intrinsically many degrees of freedom and are thus well suited for a statistical treatment.

If the euclidean quantum action is complex (corresponding to a path weighting factor in the original Minkowski functional integral which is not a pure phase) then the probabilistic interpretation and the consequent usual stochastic methods fail. A simple example of this situation is provided by a theory in which some of the coupling constants are made complex [2]. Then the functional integral can have singularities at intermediate values of the coupling constants, which are not easily studied in perturbation theory. Systems of this type include spin systems with complex temperatures or complex external fields and lattice gauge theories for complex couplings.
Another example is provided by the functional integral for quantum gravity, which is mathematically ill-defined, because of the indefiniteness of the Einstein action caused by rapidly varying conformal modes. Here it has been suggested that the integration contour over metrics should be distorted so as to include complex conformal factors [3].

Finally one of the most interesting applications is to chiral gauge theories, where the fermion determinant is not real. Very little is known about the strong coupling dynamics of these theories, although some interesting results have recently been obtained in two dimensions [4].

In a pure left-handed theory the fermion determinant, obtained after integrating over the fermion fields,

$\operatorname{det} i \emptyset_{\mathrm{L}}=\left[\operatorname{det}\left(-\not \emptyset_{\mathrm{L}}^{2}+\delta m^{2}\right)\right]^{1 / 2}$

is not real [5] because the operator

$-\not D_{\mathrm{L}}^{2}=-\mathrm{D}_{\mathrm{L}}^{2}+\frac{1}{2} g \sigma_{\mu \nu} F_{\mu \nu}$

is not hermitean. Here we have defined

$\sigma_{\mu \nu}=(1 / 2 \mathrm{i})\left[\sigma_{\mu}, \sigma_{\nu}\right], \quad \sigma_{\mu}=(1, \tau)$,

$\mathrm{D}_{\mathrm{L}}^{2}=\mathbf{D}^{2}+\mathrm{i} \mathbf{D}_{0} \tau \cdot \mathbf{D}$,

and on a lattice the fermion doubling problem can be avoided by using the second order formulation 
[6]. But since the chiral symmetry is no longer explicit the counterterm has to be fine-tuned to obtain a purely left-handed fermion. Also, while gauge invariance can be maintained in the second order lattice formulation, Lorentz invariance will be broken and is hopefully restored in the continuum limit. The fermion contribution to the action is then

$-\frac{1}{2} n_{f} \operatorname{Tr} \ln \left(-\mathrm{D}_{\mathrm{L}}^{2}+\frac{1}{2} g \sigma_{\mu \nu} F_{\mu \nu}+\delta m^{2}\right)$,

which in turn can be reexpressed in terms of purely bosonic correlations by using pseudo-fermion fields (commuting fields with the quantum number of fermions).

Let us now turn to a discussion of simple theories with complex actions. Consider a system with canonical variables $x_{1}, x_{2}, \ldots, x_{N}$ and the action $S\left(x_{1}, x_{2}, \ldots, x_{N}\right)$. Then the Langevin equation is given by $[1,7]$

$\mathrm{d} x_{i} / \mathrm{d} t=-\beta \partial S / \partial x_{i}+\eta_{i}(t)$,

where $\eta$ is a white noise satisfying

$\left\langle\eta_{i}(t)\right\rangle=0,\left\langle\eta_{i}(t) \eta_{j}\left(t^{\prime}\right)\right\rangle=2 \delta_{i j} \delta\left(t-t^{\prime}\right)$.

For real $\beta$ one can show that the time dependent probability distribution function

$P\left(x_{1}, x_{2}, \ldots, x_{N} ; t\right)$ satisfies the following

Fokker-Planck equation [8]

$\frac{\partial P}{\partial t}=\sum_{i}^{N}\left[\frac{\partial}{\partial x_{i}}\left(\frac{\partial S}{\partial x_{i}} P\right)+\frac{\partial^{2} P}{\partial x_{i}^{2}}\right]$.

It is easy to see that $P=\exp (-S)$ is a stationary solution of (7). If $S$ is nonsingular and bounded from below, it is also a stable one. Then one has

$$
\begin{aligned}
\lim _{t \rightarrow \infty} f\left(x_{1}(t), x_{2}(t), \ldots, x_{N}(t)\right) \\
=\frac{\int \prod_{i=1}^{N} \mathrm{~d} x_{i} f\left(x_{1}, x_{2}, \ldots, x_{N}\right) \mathrm{e}^{-\beta S}}{\int \prod_{i=1}^{N} \mathrm{~d} x_{i} \mathrm{e}^{-\beta S}} .
\end{aligned}
$$

For complex $\beta$, however, the relation between the left-hand side and the right-hand side of (8) is not clear, although there are some formal discussions on this point [9]. On the other hand, if one looks at the Langevin equation for a harmonic potential, i.e. $S=\frac{1}{2} x^{2}$, the solution can be worked out easily

$x(t)=\mathrm{e}^{-\beta t}\left(x_{0}+\int_{0}^{t} \mathrm{~d} \tau \eta(\tau) \mathrm{e}^{\beta \tau}\right)$.
Then it is a trivial matter to prove that, for $\operatorname{Re} \beta>0$

$\lim _{t \rightarrow \infty} x^{2 N}(t)=\frac{(2 N) !}{N !} \beta^{-N}$,

which agrees with the result obtained from the partition function. For an arbitrary action the convergence of the Langevin process to the statistical average can also be established perturbatively for large $\beta$. For example, if

$S(x)=\frac{1}{2} x^{2}+\frac{1}{4} x^{4}$,

one can show, by iterating the Langevin equation, that

$$
\begin{aligned}
& \lim _{t \rightarrow \infty} x^{2 N}(t) \\
& \quad=\frac{(2 N) !}{N !} \beta^{-N}\left[1-N(N+2) / \beta+\mathrm{O}\left(1 / \beta^{2}\right)\right],
\end{aligned}
$$

for $\operatorname{Re} \beta>0$. This suggests that for arbitrary action there may exist a region in the complex- $\beta$ plane where the Langevin process converges.

We first studied two nontrivial toy models by the Langevin equation. The first model involves the potential

$S(x)=\frac{1}{4} x^{4}$

It is easy to evaluate the following statistical averages

$\left\langle x^{2 N}\right\rangle=(4 / \beta)^{N / 2} \Gamma\left(N / 2+\frac{1}{4}\right) / \Gamma\left(\frac{1}{4}\right)$,

where $\Gamma(z)$ is the usual Gamma function. The statistical integral converges for $\operatorname{Re} \beta>0$. But the result can be continuated analytically to the entire complex- $\beta$ plane, except a cut from the origin when $N$ is odd. The Langevin equation for this action is

$\mathrm{d} x / \mathrm{d} t=-\beta x^{3}+\eta(t)$.

This equation can only be solved numerically. A simple discrete version of eq. (15) is

$x_{i+1}=x_{i}-\epsilon \beta x_{i}^{3}+\sqrt{24 \epsilon} \eta_{i}$,

where $\epsilon$ is the step size and $\eta_{i}$ is a random number in the interval $\left[-\frac{1}{2}, \frac{1}{2}\right]$. The error due to the discretization is of order of $\epsilon$. Another discrete version of eq. (15) is given by the second order 
Table 1

Results for the action $S=\frac{1}{4} \mathrm{x}^{4}$.

\begin{tabular}{|c|c|c|c|c|}
\hline \multirow{2}{*}{$\begin{array}{l}\text { Phase } \\
\text { (degrees) }\end{array}$} & \multicolumn{2}{|l|}{$\left\langle x^{2}\right\rangle$} & \multicolumn{2}{|l|}{$\left\langle x^{4}\right\rangle$} \\
\hline & exact & Langevin & exact & Langevin \\
\hline 0 & $(0.338, \quad 0.000)$ & $\left(\begin{array}{ll}0.339, & 0.000\end{array}\right)$ & $\left(\begin{array}{ll}1.000, & 0.000\end{array}\right)$ & $(1.015,0.000)$ \\
\hline 10 & $(0.337,-0.029)$ & $\left(\begin{array}{ll}0.330, & 0.028)\end{array}\right.$ & $(0.985,-0.174)$ & $(0.952,-0.167)$ \\
\hline 20 & $(0.333,-0.059)$ & $0.333,-0.061)$ & $(0.940,-0.342)$ & $0.934-0.354)$ \\
\hline 30 & $(0.326,-0.087)$ & $(0.344,-0.095)$ & $(0.866,-0.500)$ & $(0.938,-0.556)$ \\
\hline 40 & $(0.318,-0.116)$ & $(0.337,-0.125)$ & $(0.766,-0.643)$ & $0.823,-0.717)$ \\
\hline 50 & $(0.306,-0.143)$ & $0.303,-0.138)$ & $(0.643,-0.766)$ & $0.636,-0.740)$ \\
\hline 60 & $(0.293,-0.169)$ & $0.300,-0.176)$ & $(0.500,-0.866)$ & $(0.507,-0.889)$ \\
\hline 70 & $(0.277,-0.194)$ & $0.265,-0.186)$ & $(0.342,-0.940)$ & $(0.317,-0.914)$ \\
\hline 80 & $(0.259,-0.217)$ & $(0.249,-0.221)$ & $(0.174,-0.985)$ & $(0.159,-0.994)$ \\
\hline 100 & $(0.217,-0.259)$ & $(0.077,-0.289)$ & $(-0.174,-0.985)$ & $(-0.200,-1.054)$ \\
\hline 110 & $(0.194,-0.277)$ & $(-0.021,-0.303)$ & $(-0.342,-0.940)$ & $(-0.362,-0.882)$ \\
\hline 120 & $(0.169,-0.293)$ & $(-0.164,-0.331)$ & $(-0.500,-0.866)$ & $(-0.837,-1.000)$ \\
\hline 130 & $(0.143,-0.306)$ & $(-0.342,-0.125)$ & $(-0.643,-0.766)$ & $(-0.610,-1.042)$ \\
\hline 140 & $(0.116,-0.318)$ & $(-0.392,-0.031)$ & $(-0.766,-0.643)$ & $(-0.548,-0.932)$ \\
\hline 150 & $(0.087,-0.326)$ & $(-0.302,-0.123)$ & $(-0.866,-0.500)$ & $(-0.863,-0.399)$ \\
\hline 160 & $(0.059,-0.333)$ & $(-0.341,-0.131)$ & $(-0.940,-0.342)$ & $(-1.108,-0.246)$ \\
\hline 170 & $(0.029,-0.337)$ & $(-0.347, \quad 0.020)$ & $(-0.985,-0.174)$ & $(-0.980,-0.379)$ \\
\hline
\end{tabular}

Runge-Kutta equation

$x_{i+1}=\frac{1}{2}\left(x_{i+1}^{(1)}+x_{i+1}^{(2)}\right) / 2$,

with

$x_{i+1}^{(1)}=x_{i}-\epsilon \beta x_{i}^{3}+\sqrt{48 \epsilon} \eta_{i}$,

$x_{i+1}^{(2)}=x_{i+1}^{(1)}-\epsilon \beta x_{i+1}^{(1) 3}+\sqrt{48 \epsilon} \eta_{i}$.

The corresponding error for this discretization of the time derivative is of the order of $\epsilon^{2}$. We used both algorithms to evaluate the long time limit of $x^{2}$ and $x^{4}$ for this action. The results for 500000 steps are shown in table 1 . We found that the process converges for all phase angles of $\beta$, except on the imaginary axis and on the negative real axis. For $\operatorname{Re} \beta>0$ the Langevin averages converge to the value given by (14) for both $\left\langle x^{2}\right\rangle$ and $\left\langle x^{4}\right\rangle$; for $\operatorname{Re} \beta<0$ for which the statistical integral becomes meaningless the Langevin averages for $\left\langle x^{2}\right\rangle$ do not converge to the analytic continuation of (14), while they appear to do so for $\left\langle x^{4}\right\rangle$.

The second toy model is given by the action

$S(x)=\cos x$.

This action is closely related to the action of an abelian lattice gauge theory. The statistical aver- ages are given by

$$
\left\langle\cos ^{N} x\right\rangle=\left[I_{0}(\beta)\right]^{-1} \mathrm{~d}^{N} I_{0}(\beta) / \mathrm{d} \beta^{N},
$$

where $I_{\nu}(z)$ is the modified Bessel function of the first kind. The expression (20) is a meromorphic function with an infinite number of poles on the imaginary axis. The Langevin equation for this system is

$$
\mathrm{d} x / \mathrm{d} t=-\beta \sin x+\eta(t) .
$$

For large $\beta$ the solution of eq. (21) and its convergence can be established perturbatively, just like the example in eq. (11). However, for small $\beta$, the solution of eq. (21) can also be found iteratively. To the leading order in $\beta$ we have

$$
\lim _{t \rightarrow \infty} \cos x=\frac{1}{2} \beta \text {. }
$$

For arbitrary $\beta$, eq. (21) can only be solved numerically. We discretize eq. (21) as

$$
z_{i+1}=z_{i} \exp \left[-\frac{1}{2} \epsilon \beta\left(z_{i}-z_{i}^{-1}\right)+i \sqrt{24 \epsilon} \eta_{i}\right],
$$

with $z=\mathrm{e}^{\mathrm{i} x}$. It is important to note that the force term is $z-z^{-1}$ and not $z-z^{*}$, otherwise one would obtain the wrong answer.

The quantity we evaluated in the long time limit was $\cos x=\frac{1}{2}\left(z+z^{-1}\right)$. In table 2 a comparison 

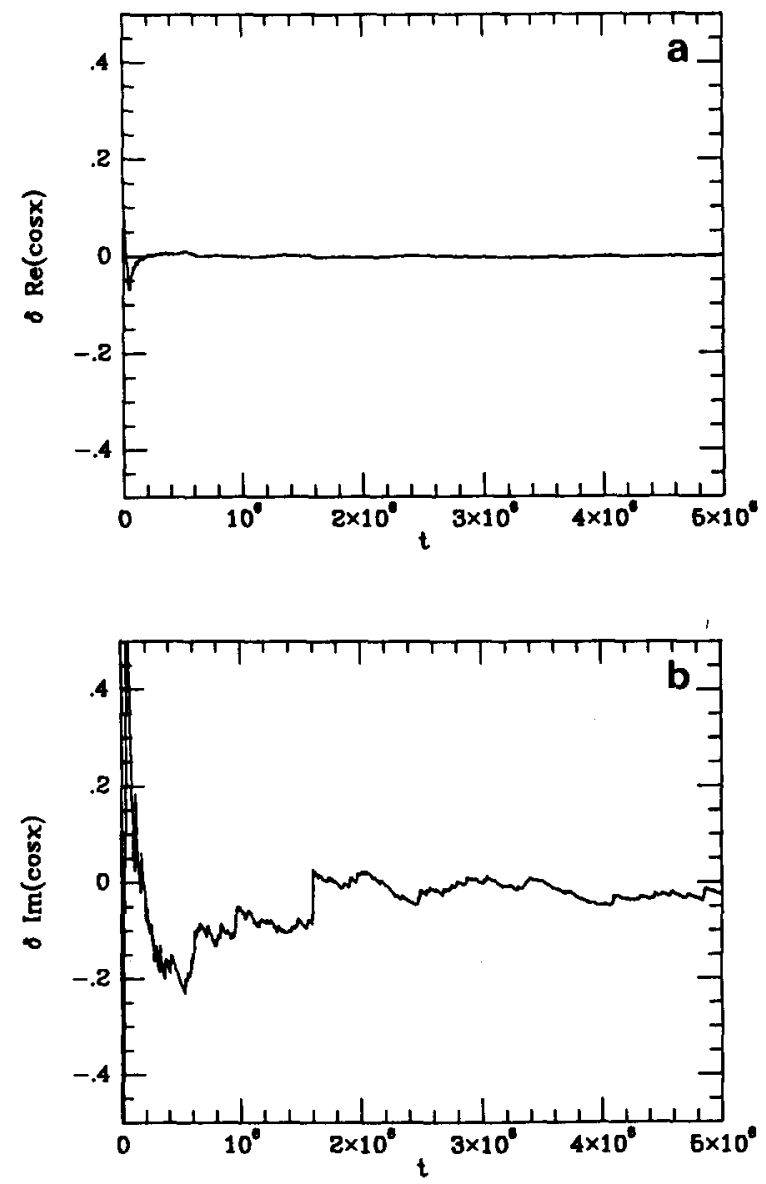

Fig. 1. The relative deviation of the Langevin average from the exact value for the action (19) at $\beta=5 \exp [\mathrm{i}(\pi / 9)]$, where $\delta x=(\langle x\rangle$ Langevin $-\langle x\rangle$ exact $) /\langle x\rangle$ exact. (a) Real part. (b) Imaginary part.

is made between the Langevin value and the value given by eq. (20). In fig. 1 we plotted the relative deviation of the numerical data from the analytic values as a function of Langevin time, for one $\beta$. For each $\beta$ we did typically 5000000 iterations. As one can see, for a fixed module of $\beta$, the convergence gets worse and worse as the phase angle of $\beta$ is increased. And also, for a fixed phase, the convergence gets better and better as the module is increased. The results are independent of the initial position, as they should be, even if one starts from a point outside the unit circle. Due to the compactness of the action, we expect convergence for $\operatorname{Re} \beta<0$. This was also observed in practice as shown in table 2 .
Table 2

Results for the action $S=\cos x$.

\begin{tabular}{lclll}
\hline$|\beta|$ & $\begin{array}{l}\phi \beta \\
\text { (degrees) }\end{array}$ & \multicolumn{2}{l}{ exact } & \multicolumn{1}{l}{ Langevin } \\
\hline 1 & 0 & $(0.446,0.000)$ & $(0.441,0.000)$ \\
1 & 10 & $(0.444,0.062)$ & $(0.464,0.037)$ \\
1 & 20 & $(0.438,0.126)$ & $(0.465,0.080)$ \\
3 & 10 & $(0.814,0.039)$ & $(0.816,0.037)$ \\
3 & 20 & $(0.827,0.076)$ & $(0.830,0.064)$ \\
3 & 30 & $(0.850,0.111)$ & $(0.846,0.099)$ \\
5 & 10 & $(0.896,0.020)$ & $(0.894,0.020)$ \\
5 & 20 & $(0.902,0.039)$ & $(0.900,0.038)$ \\
5 & 30 & $(0.912,0.056)$ & $(0.910,0.058)$ \\
5 & 45 & $(0.929,0.075)$ & $(0.925,0.078)$ \\
5 & 180 & $(-0.893,0.000)$ & $(-0.890,0.000)$ \\
\hline
\end{tabular}

As will be shown later on, these properties are also shared by the two-dimensional U(1) lattice gauge theory. Bearing in mind that there is an infinite number of poles in $\langle\cos x\rangle$ along the imaginary axis, the bad convergence might be due to the singularities which are approached as one gets closer to the imaginary axis. We also found for complex $\beta$ that when $\epsilon$ is greater than a certain value, typically 0.001 , the solution blows up, with $z$ either being trapped at the origin or starting to wander off to infinity. Thus there appear to be some instabilities introduced by the discretization. The wild fluctuation in fig. 1 is due to the fact that we have only one degree of freedom, which will improve for the two-dimensional U(1) model. We also tried the Runge-Kutta equation for this example. The fluctuation was reduced, but the improvement in the accuracy was not spectacular.

One of the simplest and soluble models in lattice gauge theory is the two-dimensional $U(1)$ gauge theory. The action for this model is given by

$S=\sum_{p}\left(1-\cos \theta_{p}\right)$,

where $p$ labels the plaquettes, and $\theta_{p}$ is the phase angle of the $p$ th plaquette. The average action per plaquette is given by

$\langle S\rangle=1-I_{1}(\beta) / I_{0}(\beta)$.

It is again a meromorphic function with an infinite number of poles along the imaginary $\beta$ axis. For complex $\beta$ we assign an arbitrary complex number 

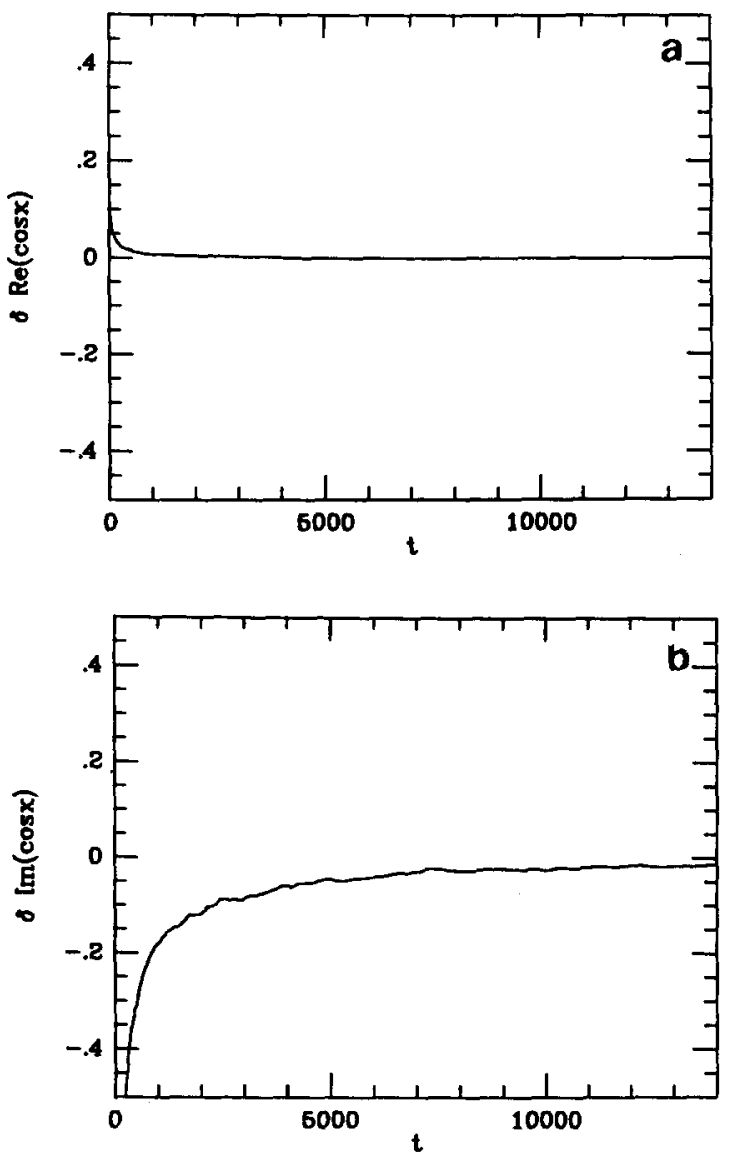

Fig. 2. The relative deviation of the Langevin average from the exact value for the action (24) at $\beta=5 \exp [\mathrm{i}(\pi / 9)]$. (a) Real part. (b) Imaginary part.

$z_{i j}$ (it need not be a pure phase) to each link $l_{i j}$ with the property $z_{i j}=z_{j i}^{-1}$. For each plaquette $p_{i j k l}$ we have, then, $z_{p}=z_{i j} z_{j k} z_{k l} z_{l i}$. The action then becomes

$S=\sum_{p}\left[1-\frac{1}{2}\left(z_{p}+z_{p}^{-1}\right)\right]$.

The Langevin process is given by the following iterative equation [10]:

$$
\begin{aligned}
& z_{l(i+1)}=z_{l(i)} \\
& \quad \times \exp \left\{\frac{1}{2} \epsilon \beta\left(\zeta_{1}+\zeta_{2}\right)\left[z_{l(i)}-\left(\zeta_{1} \zeta_{2} z_{l(i)}\right)^{-1}\right]\right. \\
& \left.\quad+\mathrm{i} \sqrt{24 \epsilon} \eta_{l(i)}\right\} .
\end{aligned}
$$

Using this procedure we studied a two-dimensional square lattice of size $20 \times 20$. As shown in fig. 2 , the result is very similar to that of the action in eq. (19). In other words, for a larger and larger module of $\beta$, the convergence gets better and better. We also observed that if the step size $\epsilon$ is too large the solution blows up.

Presumably, these properties are shared by $\mathrm{U}(N)$ lattice gauge theories. It is straightforward to generalize the Langevin algorithm to nonabelian gauge theories. What one should do is to assign an arbitrary complex matrix $U_{i j}$ to each link $l_{i j}$, and replace all $U_{i j}^{\dagger}$ by $U_{i j}^{-1}$. For the $\operatorname{SU}(N)$ group one should ensure that $\operatorname{det} U_{i j}=1$. Then the matrices can never become singular. So the procedure might be more stable than for the $\mathrm{U}(N)$ group.

This work was supported by the US Department of Energy under contract no. DE-AC0276ER02220.

\section{References}

[1] G. Parisi and Y.-S. Wu, Sci. Sinica 24 (1981) 483; J.R. Klauder, Stochastic Quantization, Lectures XXII Schladming school, Bell Labs preprint (March 1983).

[2] G. Parisi, Phys. Lett. 131B (1983) 393.

[3] S.W. Hawking, in: General relativity-an Einstein centenary survey, eds. S.W. Hawking and W. Israel (Cambridge U.P., London, 1979).

[4] R. Jackiw and V. Ramarajan, Phys. Rev. Lett. 54 (1985) 1219.

[5] G. Parisi and Y.-C. Zhang, Phys. Lett. 131B (1983) 149.

[6] R.P. Feynman, Phys. Rev. 84 (1951) 108; R.P. Feynman and M. Gell-Mann, Phys. Rev. 109 (1958) 193.

[7] G. Parisi, Nucl. Phys. B180 [FS2] (1981) 378; B205 [FS5] (1982) 337.

[8] I. Guikhman and A. Skorokhod, Introduction à la Théorie des Processes Aleatoires (Mir, Moscow, 1980).

[9] J.R. Klauder and W.P. Petersen, Bell Labs preprint (December 1984).

[10] M. Falcioni et al., Nucl. Phys. B215 [FS7] (1983) 265; H.W. Hamber and U.M. Heller, Phys. Rev. D29 (1984) 928;

A.A. Migdal, M.I. Polikarpov, A.I. Veselov and V.P. Yurov, Phys. Lett. 135B (1984) 145, and references therein. 